\title{
Pseudoangiomatous hyperplasia of mammary stroma associated with gynaecomastia
}

\author{
M F G Milanezi, F P Saggioro, S G Zanati, R Bazan, F C Schmitt
}

\begin{abstract}
Aims-To evaluate the prevalence of pseudoangiomatous hyperplasia of mammary stroma in gynaecomastia and its immunohistochemical profile in this setting.

Methods-Eighty eight cases of gynaecomastia recovered from the files of the department of pathology, Botucatu School of Medicine from 1976 to 1996 were studied. In the cases associated with pseudoangiomatous hyperplasia of mammary stroma, immunoreactivity for cytokeratins (CAM 5.2), vimentin, CD34, factor VIII related antigen, and the oestrogen and progesterone receptors were studied. Results-Pseudoangiomatous hyperplasia of mammary stroma was found in 21 of 88 cases of gynaecomastia $(23.8 \%)$. In all cases, the cells lining the spaces were positive for vimentin, whereas CAM 5.2 and factor VIII related antigen were consistently negative. Nineteen of the 21 cases showed immunoreactivity for CD34. Ductal epithelial cells were positive for both the oestrogen receptor and the progesterone receptor, whereas stromal cells were negative.

Conclusions-Pseudoangiomatous hyperplasia of mammary stroma was present in approximately one quarter of the cases of gynaecomastia. This immunohistochemical study confirms the mesenchymal origin of the stromal cells that line the pseudovascular spaces, as has been found in female cases of pseudoangiomatous hyperplasia of mammary stroma.
\end{abstract} (f Clin Pathol 1998;51:204-206)

The Department of logy, Botucat School of Medicine, UNESP, Botucatu, São Paulo 18600, Brazil M F G Milanezi F P Saggioro

S G Zanati

R Bazar

The Unit of Molecular Pathology, Institute of Pathology and Molecular Immunology of the University of Porto (IPATIMUP), 4200 Porto, Portugal

F C Schmitt

Correspondence to: Dr F C Schmitt, IPATIMUP, Rua Roberto Frias S/N, 4200 - Porto, Portugal. email: fernando.schmitt@ hook.ipatimup.up.pt

Accepted for publication 9 December 1997 neoplastic lesions or, more rarely, as a palpable mass. The aetiology of pseudoangiomatous hyperplasia of mammary stroma remains unknown, ${ }^{1}$ despite speculations about the influence of hormones, especially progesterone, on its origin. The purpose of this study was to evaluate the presence of pseudoangiomatous hyperplasia of mammary stroma in cases of gynaecomastia and to determine its immunohistochemical profile in this setting.

\section{Methods}

Eighty eight cases of gynaecomastia were collected consecutively from the files of the Department of Pathology, Botucatu School of Medicine between 1976 and 1996. The surgical specimens were fixed in formalin, embedded in paraffin wax, sectioned at $5 \mu \mathrm{m}$ thickness, and stained with haematoxylin and eosin. Clinical histories were obtained from the surgical reports. All cases were evaluated independently by three of us (MFGM, FPS, and FCS) to confirm the diagnosis of gynaecomastia and to detect pseudoangiomatous hyperplasia of mammary stroma. Pseudoangiomatous hyperplasia of mammary stroma had been recognised previously in only one case. In addition to pseudoangiomatous hyperplasia of mammary stroma, other histological parameters such as ductal hyperplasia, lobule formation, stromal fibrosis, and association with carcinoma were also evaluated. Immunohistochemistry was performed on the cases in which there was an association between gynaecomastia and pseudoangiomatous hyperplasia of mammary stroma. It was performed on formalin fixed, paraffin wax embedded tissue using the avidinbiotin complex technique (ABC) with diaminobenzidine (DAB) as chromogen, as previously described by our group. ${ }^{6}$ Antigen retrieval was performed using microwave pretreatment. The monoclonal antibodies used were specific for: vimentin (Dakopatts, Glostrup, Denmark; diluted $1 / 80$ in $5 \%$ bovine serum albumin (BSA)/Tris buffered saline (TBS)), low molecular weight keratin-CAM 5.2 (Becton-Dickinson, Erembodegem, Belgium; diluted $1 / 5$ in $5 \% \mathrm{BSA} / \mathrm{TBS}), \mathrm{CD} 34$ (Dakopatts; diluted $1 / 20$ in $5 \% \mathrm{BSA} / \mathrm{TBS}$ ), factor VIII related antigen (Dakopatts; diluted $1 / 60$ in $5 \%$ BSA/TBS), the oestrogen receptor (Novocastra, Newcastle, UK; diluted 1/60 in $5 \% \mathrm{BSA} / \mathrm{TBS}$ ), and the progesterone receptor (Immunotech, Marseille, France; diluted 1/40 in $5 \% \mathrm{BSA} / \mathrm{TBS})$. Statistical analysis was performed using the $\chi^{2}$ test with Yates's correction. Values of $p<0.05$ were considered to be statistically significant.

Results

Eighty eight cases of gynaecomastia were obtained over a period of 20 years. The age range was 10-74 years (mean, 42 years). 


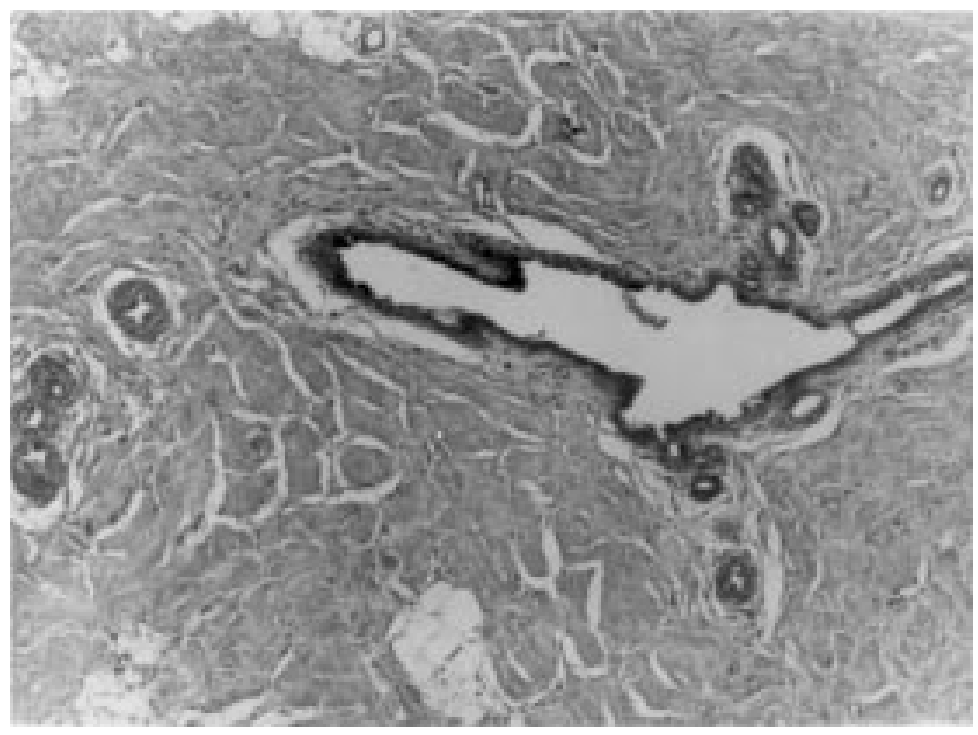

Figure 1 Pseudoangiomatous hyperplasia of mammary stroma in a case of gynaecomastia. Note the empty pseudovascular spaces lined by spindle cells. Haematoxylin and eosin stained.

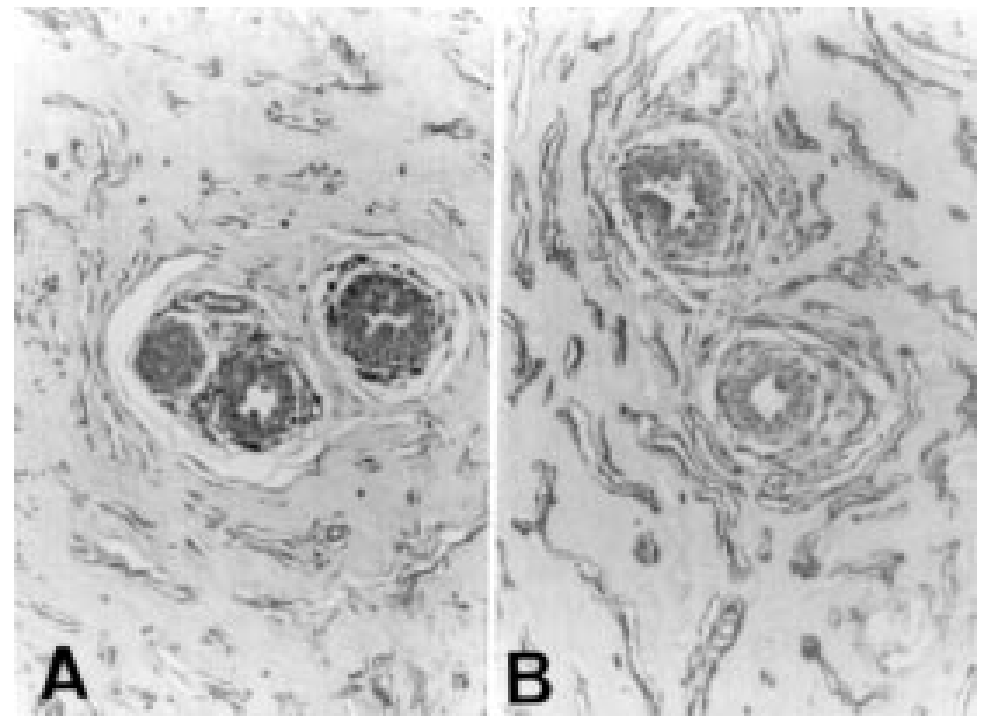

Figure 2 Immunohistochemical staining showing that the spindle cells lining the spaces are immunoreactive for $(A)$ vimentin and $(B) C D 34$.

Twenty one cases (23.8\%) showed typical microscopical features of pseudoangiomatous hyperplasia of mammary stroma, characterised by a complex pattern of anastomosing empty spaces in a dense, keloid-like stroma (fig 1). These spaces were lined by spindle cells. The immunohistochemical studies showed positivity of the cells for vimentin in all cases and for CD34 in nineteen cases (90.4\%) (fig 2). Immunoreactivity to cytokeratins, factor VIII related antigen, or hormone receptors (oestrogen and progesterone) was not detected. In one

Table 1 Comparative analysis of age and morphological findings in gynaecomastia associated and non-associated with pseudoangiomatous hyperplasia of mammary stroma (PHMS)

\begin{tabular}{llll}
\hline Clinicopathological findings & $\begin{array}{l}\text { Gynaecomastia with } \\
\text { PHMS }(n=21)\end{array}$ & $\begin{array}{l}\text { Gynaecomastia without } \\
\text { PHMS ( } n=67)\end{array}$ & p value \\
\hline Age (10-40 year old) & $95 \%(20$ cases $)$ & $74.6 \%(50$ cases $)$ & 0.04 \\
Ductal hyperplasia without atypia & $71.4 \%(15$ cases $)$ & $29.0 \%(20$ cases $)$ & 0.0007 \\
Stromal fibrosis & $9.5 \%(2$ cases $)$ & $19.4 \%(13$ cases $)$ & 0.29 \\
Lobule formation & $24 \%$ (5 cases) & $7.5 \%$ (5 cases $)$ & 0.03 \\
\hline
\end{tabular}

case, an equivocal positivity for the progesterone receptor in the spindle cells lining the pseudoangiomatous spaces was seen. Ductal epithelial cells were positive for the oestrogen receptor, the progesterone receptor, and cytokeratin. The analysis of age and morphological findings in relation to the association or non-association of pseudoangiomatous hyperplasia of mammary stroma with gynaecomastia is summarised in table 1 . Patients with gynaecomastia associated with pseudoangiomatous hyperplasia of mammary stroma were younger than patients without pseudoangiomatous hyperplasia of mammary stroma $(p=0.04)$. Ninety five per cent of the cases of gynaecomastia with pseudoangiomatous hyperplasia of mammary stroma were aged between 10 and 40 years, with 14 (67\%) patients aged between 10 and 24 years. Epithelial hyperplasia and lobule formation were observed in $71.4 \%$ and $24 \%$ of the 21 cases of gynaecomastia with pseudoangiomatous hyperplasia of mammary stroma, respectively. In contrast, epithelial hyperplasia was present in only $29 \%$ of the 67 cases of gynaecomastia without pseudoangiomatous hyperplasia of mammary stroma. In addition, stromal fibrosis was more common in this group (19.4\%) than in the group with pseudoangiomatous hyperplasia of mammary stroma, although this was not statistically significant. Ductal epithelial hyperplasia with atypia and carcinoma were not found in any of the cases.

\section{Discussion}

Enlargement of the male breast is caused by gynaecomastia in more than $85 \%$ of cases. ${ }^{57}$ Gynaecomastia is often associated with metabolic and endocrine disorders that induces direct or indirect changes in the metabolism of steroids that stimulate cell proliferation in male glandular breast tissue. ${ }^{7}$ Progressive changes are observed with time, independent of the aetiology; therefore, the microscopical appearance of gynaecomastia depends on the duration of the disease. ${ }^{8}$ The development of an oedematous periductal stroma in association with fibroblastic and ductal proliferation as well as epithelial hyperplasia, probably indicates the very early response of male breast tissue to mammotropic stimuli. Later on, an increasing density of periductal stroma can be observed followed by progressive fibrosis and hyalinisation. In association with epithelial atrophy, this phenomenon may be regarded as the terminal phase of the process. Pseudoangiomatous hyperplasia of mammary stroma was first described in 1986 by Vuitch et al as a breast lesion that simulated a vascular tumour. ${ }^{9}$ They also noted that small foci of pseudoangiomatous hyperplasia of mammary stroma were common in hyperplastic breast tissue from premenopausal women or during the luteal phase of the menstrual cycle. Traditionally, the mammary stroma is divided into an intralobular component that is hormone dependent, and an interlobular component that is relatively unresponsive to hormones. The lobular stroma and the epithelium change during the menstrual cycle. The lobulocentric pattern seen in 
pseudoangiomatous hyperplasia of mammary stroma is similar to lobular stromal changes in the luteal phase of the menstrual cycle. Thus, pseudoangiomatous hyperplasia of mammary stroma could be the result of intralobular stromal expansion. ${ }^{3}$ Some authors believe that pseudoangiomatous hyperplasia of mammary stroma is an exaggerated response to progesterone in oestrogen primed tissue. Anderson et al showed expression of progesterone receptors in lining cells of the pseudovascular spaces in cases observed in female breast tissue. ${ }^{10}$ Although pseudoangiomatous hyperplasia of mammary stroma has already been described in male breast tissue, ${ }^{4}$ there are no studies concerning the influence of hormones on the development of pseudoangiomatous hyperplasia of mammary stroma in male breast tissue, nor any studies that we are aware of concerning its immunohistochemical profile in this setting. Some of our results suggest that hormones might play a role in the induction of pseudoangiomatous hyperplasia of mammary stroma in gynaecomastia. ${ }^{11}$ The frequent presence of ductal epithelial hyperplasia and lobule formation in cases of gynaecomastia associated with pseudoangiomatous hyperplasia of mammary stroma and the younger age of the patients favours this possibility, in spite of the apparent lack of hormonal receptors in the stromal cells. With regard to the differentiation of the stromal cells, the negativity for factor VIII related antigen excludes their endothelial origin. CD34, identified as a marker for haemopoietic progenitor cells in the bone marrow, stains a variety of tissue components including endothelial cells, dermal dendritic cells, and fibroblasts/myofibroblasts. The strong positivity of the stromal cells for CD34 and vimentin confirms the probable fibroblastic/myofibroblastic origin of the pseudoangiomatous hyperplasia. ${ }^{3}$ A remarkable feature of this lesion is the distribution of the cells around an empty space, characterising an unusual fibroblastic/myofibroblastic polarisation. The presence of these spaces in frozen sections refutes the possibility that they are just an artefact. Ultrastructural studies can contribute to a better understanding of the pathogenesis and morphological basis of this lesion.
In summary, our study showed that pseudoangiomatous hyperplasia of mammary stroma is present in about a quarter of the cases of gynaecomastia. This lesion was more frequent in younger patients and was frequently associated with cases displaying ductal hyperplasia and lobule formation, thus suggesting the involvement of hormonal stimuli in its pathogenesis. These findings are in accord with those of Badve and Sloane, ${ }^{1}$ who demonstrated a strong positive association between pseudoangiomatous hyperplasia of mammary stroma and the so called initial and intermediate phases of gynaecomastia, where epithelial proliferation is predominant. The immunohistochemical results confirm the fibroblastic mesenchymal origin of the stromal cells, as described in the female breast.

We thank Professor Manuel Sobrinho-Simões (Unit of Molecular Pathology, IPATIMUP, Porto University) for his helpful advice in the preparation and revision of the manuscript. This work was partly supported by a research grant from the Fundação para a Ciência e Tecnologia, Portugal (PRAXIS XXI BCC/6445/95)

1 Badve S, Sloane JP. Pseudoangiomatous hyperplasia of male breast. Histopathology 1995;26:463-6.

2 Ibrahim RE, Sciotto CG, Weidner N. Pseudoangiomatous hyperplasia of mammary stroma-some observations regarding its clinicopathologic spectrum. Cancer 1989;63: $1154-60$.

3 Powell CM, Cranor ML, Rosen PP. Pseudoangiomatous stromal hyperplasia. A mammary stromal tumor with myofibroblastic differentiation. Am F Surg Pathol 1995;19: $270-7$.

4 Seidman JD, Borkowisky A, Aisner SC, et al. Rapid growth of pseudoangiomatous hyperplasia of mammary stroma in axillary gynecomastia in an immunosuppressed patient. Arch Pathol Lab Med 1993;117:736-8

5 Hunfeld KP, Bassler R, Kronsbein H. "Diabetic mastopaHunfeld KP, Bassler R, Kronsbein H. "Diabetic mastopa-
thy" in the male breast-a special type of gynecomastia. thy" in the male breast-a special
Pathol Res Pract 1997;193:197-205.

6 Schmitt FC, Ferreira MP. MIB-1 is a suitable marker of proliferative activity in formalin-fixed, paraffin-embedded sections of breast cancer. Int F Surg Pathol 1995;2:287-94.

7 Williams MJ. Gynecomastia. Its incidence, recognition and host characteristics in 447 autopsy cases. Ann F Med 1963; 34:103-12.

8 Nicolis GL, Modlinger RS, Gabrilove JL. A study of the histopathology of human gynecomastia. $\mathcal{F}$ Clin Endocrinol 1971;32:173-8.

9 Vuitch MF, Rosen PP, Erlandson RA. Pseudoangiomatous hyperplasia of mammary stroma. Hum Pathol 1986;17: 185-91.

10 Anderson C, Ricci A, Pedersen CA, et al. Immunocytochemical analysis of estrogen and progesterone receptors in benign stromal lesions of the breast-evidence for hormonal etiology in pseudoangiomatous hyperplasia of mammary stroma. Am $\mathcal{F}$ Surg Pathol 1991;15:145-9.

11 Bonnayan GA, Hajdu SI. Gynecomastia. Clinicopathologic study of 351 cases. Am 7 Surg Pathol 1972;57:431-7. 